\title{
POLICY Is it appropriate to link 'old age' to certain causes of death on the medical certificate of cause of death?
}

\author{
Authors: Vedamurthy Adhiyaman ${ }^{\mathrm{A}}$ and Indrajit Chattopadhyay ${ }^{\mathrm{A}}$
}

\begin{abstract}
In the current list of acceptable causes of death in the medica certificate of cause of death, some causes are either linked to old age or could be used for all people over the age of 80 years. Notable ones include 'frailty of old age', 'debility of old age' and 'senility'. It is best to avoid these terms as they provide an erroneous impression that these conditions occurred because a person was old; we recommend that the list of acceptable causes of death is revised by engaging all stakeholders.
\end{abstract}

KEYWORDS: MCCD, frailty, old age, senility, debility

DOI: $10.7861 /$ fhj.2021-0050

\section{Introduction}

It is crucial to give an accurate cause death as far as possible in the medical certificate of cause of death (MCCD). The list of acceptable causes of death was updated last year by the Royal College of Pathologists. 'Even though 'old age' has been as an acceptable cause of death for a long time, the list also has other causes linked to the term 'old age' that includes 'frailty of old age' and 'debility of old age'. Also, terms like 'advancing years', 'senility', 'senile dementia' and 'old age' could be used if a person is above the age of 80 years. However, these terms are not used in clinical practice and there has been some criticism recently regarding the use of 'frailty of old age' in the MCCD, especially when the family thought the person wasn't frail. ${ }^{2}$ Hence, to gather the views of geriatricians, we undertook an online survey regarding the appropriateness of use of some of these causes of death in the MCCD.

\section{Methods}

We devised an online questionnaire comprising nine questions and one free-text box. The questionnaire was sent to the Sarcopenia and Frailty Research Special Interest Group (SIG) of the British Geriatrics Society and to a list of consultant geriatricians via the authors' personal contacts.

Authors: ${ }^{\text {A }}$ consultant geriatrician, Glan Clwyd Hospital, Rhyl, UK

\section{Results}

There were 69 respondents to the survey, of which, eight were from the SIG (Table 1). The majority (73.9\%) of the respondents felt that 'frailty of old age' should not be used as a cause of death and that this should be referred to as just 'frailty'. Seventy per cent felt that 'frailty of old age' is ageist terminology and $90 \%$ disagreed that this term could be applied to all persons above the age of 80 years. Almost all disagreed that 'debility of old age' and 'senility' should be acceptable as causes of death in the MCCD. There was no difference in opinion between the SIG members and other geriatricians. Selected comments from the free-text box expressing different views are given in Box 1.

\section{Discussion}

The results from our survey demonstrate that causes of death should not be linked to old age. This is probably because it implies that these conditions occur only in people above the age of 80 years and people develop these conditions simply because they are old.

Even though few other conditions (including senile dementia and old age) were listed as causes that could be used if a person was above the age of 80 years, we wanted to explore certain causes that are specifically linked to the term 'old age' that erroneously give an impression that they only occur because a person is aged above 80 years.

One-tenth of those surveyed had faced some concerns relating to 'frailty of old age' documented in the MCCD from families of the deceased. Frailty is a state of impaired resolution to homoeostasis following a stressor event and is not exclusively associated with old age. ${ }^{3}$ Even though it is common when people get older, not every person who is older than 80 years becomes frail, and frequently we encounter people younger than 80 years who are frail. Even though the majority of people who become frail have associated multimorbidities, some develop frailty in the absence of any life-threatening illnesses. 3,4 However, the true incidence of frailty purely linked to old age is unclear.

Ninety per cent disagreed that 'debility of old age' should be an acceptable cause and $99 \%$ disagreed that 'senility' should be on the list of acceptable causes of death for the MCCD. Since sarcopenia is often linked with frailty, about half felt that this could be used as a cause of death in the MCCD.

The recent guidance from the Office for National Statistics (ONS) discourages using 'old age' or 'frailty of old age' as the sole cause of death, unless only in very limited circumstances. ${ }^{5}$ If 


\section{Table 1. Online questionnaire, $n=69$}

\section{Question}

Are you a member of Sarcopenia and Frailty Research Special Interest Group?

Do you think that the term 'frailty of old age' is an ageist terminology?

Are you aware that 'frailty of old age' (for people aged above 80 years) is in the list of acceptable causes of death?

Would you agree that this could be applied to all people who are above the age of 80 years?

Do you think we should use the term 'frailty' rather than 'frailty of old age' in the death certificate?

Have you had any concerns from anyone when you specifically used the term 'frailty of old age' on the death certificate?

Do you think 'sarcopenia' should be included in the list of causes of death?

Do you think 'debility of old age' (applicable for people aged over 80 years) should be on the list of acceptable causes of death?

Do you think 'senility' (applicable for people aged over 80 years) should be on the list of acceptable causes of death?

\section{Yes, $\% \quad$ No, $\% \quad$ Maybe / not} applicable, \%

$11.6 \quad 88.4$

$69.6 \quad 30.4$

$82.6 \quad 17.4$

$8.7 \quad 91.3$

26.1

$11.6 \quad 52.2 \quad 36.2$ (not applicable)

$10.1 \quad 43.5 \quad 46.4$ (maybe)

$10.1 \quad 89.9$

1.4

98.6

\section{Box 1. Selected free-text comments from the questionnaire}

\section{Frailty:}

Using 'frailty of old age' conflates two separate terms. Frailty is not age specific and old age can end up being a surrogate for frailty. Frailty can occur in those younger than 80 [years] and not every person over the age of 80 [years] has frailty, so the terminology is inaccurate at present.

'Frailty of old age' is an appropriate term that can be used in death certification.

I hate the word 'frailty'. When we write [it] in the death certificate, then we are missing an important and treatable underlying medical condition.

'Frailty of old age' is a potentially useful descriptor for a small group of patients who are extremely frail with an associated general decline but no obvious reversible final cause

'Advanced frailty' should also be included as an acceptable cause of death.

I had concerns raised from the deceased's family on use of the term 'frailty' in [the] MCCD as the family was not aware that their relative was 'frail' and this condition was never mentioned to them by any other clinician before.

I do think that 'frailty of old age' is a useful term, particularly when it denotes a slow decline [to] death without a clear pathology.

Frailty is not age related. Frailty depends on comorbidities, physiological reserve, disease burden and support.

\section{Debility and senility:}

I don't like debility as this may be a reversible condition and is not necessarily progressive with appropriate intervention.

Senility/debility is less acceptable to families and could be considered ageist.

'Senility of old age' is a term that means little.

Debility and, definitely, senility are not acceptable.

Senility sounds pejorative. Sarcopenia is such a specialist word it will be misinterpreted and misused.

Debility sounds vague and probably doesn't have the supporting evidence base (unlike frailty).

\section{General comments:}

We should not use any terms linked to old age. This gives a wrong impression that old age causes the condition.

'Old age' [can be used] if [the] patient is biologically older and has no other cause of death.

'Old age' alone can be used; it is what I want written on my death certificate.

I would prefer the term 'older' age.

Your questions feel weighted or biased towards the negative view of the term.

As the geriatric population is ever increasing and 80 [years] is no longer old in a relative sense, we should refrain from age-related descriptions, if possible.

Before formal decisions are taken on this topic, it should be discussed with a group of patient representatives (and/or their families).

[It's a] very emotive issue as [the] family sees the death certificate, we can't deny that old age exists and this does not make us ageist to say this but have to ensure what we put is not going to upset the family.

I think the aim is to steer away from defining any disability on grounds of chronological 'age' alone.

$\left[I^{\prime} m\right]$ interested to see results of this.

MCCD $=$ medical certificate of cause of death 
these are used as causes contributing to death, there should be adequate information in the MCCD to justify this. Many trusts and health boards in the UK now have the medical examiner service to scrutinise all deaths, and it would be prudent for them to question the validity and accuracy of some of these diagnoses if a practitioner chooses to include them in the MCCD.

We contacted the ONS to find out the frequency of use of the term 'frailty of old age', but we were informed that there isn't a specific code for 'frailty' or 'old age' in the globally used diagnostic tool (the International Statistical Classification of Diseases and Related Health Problems, 10th revision (ICD-10)), so this could not be easily explored from their database (personal communication). ${ }^{6}$ Instead, terms such as 'old age' and 'frailty' are either coded as R54 (a code for 'age-related physical debility' or 'senility') and R68.8 (a code for 'other specified general symptoms and signs') in ICD-10.

For the years 2017, 2018 and 2019, there were 7,661, 8,038 and 7,414 deaths coded under R54, respectively, and 3,310, 4,441 and 4,781 under R68.8 for England and Wales, respectively (personal communication). ${ }^{7}$ The increase in the number of death codes as R68.8 might reflect increase in use of terms such as frailty or old age.

Hawley's paper looking at the usage of 'old age' as a cause of death on 4,300 cremation papers revealed that 300 (7\%) deaths were stated to be associated with old age and 98 were attributed to old age alone. ${ }^{8}$ Hawley's further enquiries revealed between one and six comorbid conditions in at least two-thirds of those certified as dying solely from 'old age' and only $8 \%$ had no underlying medical conditions.

A limitation of our study is the small number of respondents, and one respondent mentioned that the way the questions were phrased might have influenced the answers. Our low response rate is, however, in keeping with the evidence that responses to webbased surveys are decreasing continuously. ${ }^{9}$

\section{Conclusion}

We realise the importance of recognising frailty as a syndrome in appropriate patients. However, if this is deemed as a cause of death then it should be documented in the MCCD as 'frailty syndrome' rather than 'frailty of old age', and the conditions leading to frailty should be clearly stated. Failure to adequately record important comorbid conditions would not only affect the statistics of the occurrence of these medical conditions but would also affect health policies and investment on managing these conditions.

Other terms like 'debility of old age' and 'senility' should be removed from the list of acceptable causes of death in the MCCD. Moreover, debility and senility are not clinically used terms and they are arcane, emotive and may also be offensive to the bereaved. The current list of acceptable causes of death needs a thorough review not only because of some of these terms are linked to old age, but also as there are many other diagnoses and conditions in that list that require clarification too. However, this revision needs to be undertaken through a constructive mechanism by engaging all the stakeholders, namely the Royal College of Pathologists, patient representatives, clinicians, registrars, medical examiners and coroners.

\section{Conflicts of interest}

Dr Vedamurthy Adhiyaman also has a role as a medical examiner.

\section{References}

1 Lishman S. Cause of death list. The Royal College of Pathologists, 2020. www.rcpath.org/uploads/assets/c16ae453-6c63-47ff8c45fd2c56521ab9/G199-Cause-of-death-list.pdf [Accessed 26 February 2021].

2 Relatives \& Residents Association. 'Frailty' and death certificates: worrying trends. R\&RA. www.relres.org/frailty-and-death-certificates [Accessed 27 February2021].

3 Hanlon P, Nicholl BI, Jain BD et al. Frailty and pre-frailty in middle aged and older adults and its association with multimorbidity and mortality: a prospective analysis of 493737 UK Biobank participants. Lancet Public Health 2018;3:e323-32.

4 Fulop T, Larbi A, Witkowski JM. Aging, frailty and age-related diseases. Biogerontology 2010;11:547-63.

5 Office for National Statistics. Guidance for doctors completing Medical Certificates of Cause of Death in England and Wales. ONS. https://assets.publishing.service.gov.uk/government/uploads/ system/uploads/attachment_data/file/877302/guidance-fordoctors-completing-medical-certificates-of-cause-of-deathcovid-19.pdf [Accessed 26 February 2021].

6 World Health Organization. International Statistical Classification of Diseases and Related Health Problems, 10th revision. WHO, 2019.

7 Nomis office labour market statistics. Mortality statistics - underlying cause, sex and age. Office for National Statistics, 2020. www. nomisweb.co.uk/query/construct/summary.asp?mode=construct\& version $=0 \&$ dataset $=161$ [Accessed 27 April 2021].

8 Hawley CL. Is it ever enough to die of old age? Age Ageing 2003:32:484-6.

9 Fan W, Yan Z. Factors affecting response rates of the web survey: A systematic review. Computers in Human Behavior 2010;26:132-9.

Address for correspondence: Dr Vedamurthy Adhiyaman, Glan Clwyd Hospital, Rhuddlan Road, Bodelwyddan, Rhyl LL18 5UJ, UK.

Email: vedamurthy.adhiyaman@wales.nhs.uk

Twitter: @adhiyaman 